Mathematical Modelling and Analysis

Volume 5, 2000, PAGeS 76-85

(C) 2000 Technika

\title{
TWO-GRID ITERATION METHOD FOR WEAKLY SINGULAR INTEGRAL EQUATIONS
}

\author{
K. HAKK, A. PEDAS ${ }^{1}$ \\ Institute of Applied Mathematics, University of Tartu \\ J. Liivi 2, 50409 Tartu, Estonia \\ E-mail: arvet.pedas@ut.ee, kristiina.hakk@ut.ee
}

Received October 10, 1999

\begin{abstract}
For the solution of weakly singular integral equations by the piecewise polynomial collocation method it is necessary to solve large linear systems. In the present paper a two-grid iteration method for solving such systems is constructed and the convergence of this method is investigated.
\end{abstract}

\section{INTRODUCTION}

Consider the linear integral equation

$$
u(t)=\int_{0}^{b} K(t, s) u(s) d s+f(t), \quad 0 \leq t \leq b,
$$

where $b>0$ and $f:[0, b] \rightarrow \mathbb{R}$ is a given continuous function. Throughout this paper we shall suppose that the kernel $K$ has the form

$$
K(t, s)=a(t, s) \kappa(t-s)
$$

where

\footnotetext{
${ }^{1}$ This research was supported by the Estonian Science Foundation, grant No. 2999.
} 
(A1) the function $\kappa(\tau)$ is $m-1$ times $(m \geq 1)$ continuously differentiable with respect to $\tau$ for $\tau \in[-b, b] \backslash\{0\}$ and such that the estimates

$$
\left|\kappa^{(k)}(\tau)\right| \leq b_{k}|\tau|^{-\alpha-k}, \quad k=0,1, \ldots, m-1,
$$

hold with $0<\alpha<1$ and some positive constants $b_{0}, b_{1}, \ldots, b_{m-1}$ for all $\tau \in[-b, b] \backslash\{0\} ;$

(A2) the function $a(t, s)$ is $m$ times continuously differentiable on $[0, b] \times[0, d]$ and $[0, b] \times[d, b]$, where $d$ is a fixed point in the interval $(0, b)$.

Let $C^{k}(X)$ denote the space of $k$ times continuously differentiable functions $x: X \rightarrow \mathbb{R}, X \subset \mathbb{R} \equiv(-\infty ; \infty), C(X)=C^{0}(X)$, and set

$$
\begin{gathered}
E^{\alpha, m} \equiv\left\{u \in C[0, b] \cap C^{m}(0, d) \cap C^{m}(d, b):\right. \\
\left.\sup _{\substack{0<t<b \\
t \neq d}} \frac{\left|u^{(m)}(t)\right|}{t^{-(\alpha+m-1)}+|t-d|^{-(\alpha+m-1)}+(b-t)^{-(\alpha+m-1)}}<\infty\right\} ;
\end{gathered}
$$

$E^{\alpha, m}$ is a Banach space under the norm

$$
\|u\|_{E^{\alpha, m}}=\max _{0 \leq t \leq b}|u(t)|+\sup _{\substack{0<t<b \\ t \neq d}} \frac{\left|u^{(m)}(t)\right|}{t^{-(\alpha+m-1)}+|t-d|^{-(\alpha+m-1)}+(b-t)^{-(\alpha+m-1)}} .
$$

Note that $C^{m}[0, b] \subset E^{\alpha, m}$. It follows from $u \in E^{\alpha, m}$ that $u \in C[0, b] \cap$ $C^{m}(0, d) \cap C^{m}(d, b)$ and the estimates

$$
\left|u^{(k)}(t)\right| \leq c_{k}\left[t^{-(\alpha+k-1)}+|t-d|^{-(\alpha+k-1)}+(b-t)^{-(\alpha+k-1)}\right], k=1, \ldots, m,
$$

hold with some positive constants $c_{1}, \ldots, c_{m}$ for $0<t<d$ and $d<t<b$.

The following result (see $[11,12,8]$ ) characterizes the regularity properties of solutions of equation (1.1).

Lemma 1.1. Let the assumptions (A1) and (A2) about the kernel (1.2) hold, and let $f \in E^{\alpha, m}$. If integral equation (1.1) has an integrable solution $u \in$ $L^{1}(0, b)$ then $u \in E^{\alpha, m}$.

Remark 1.1. If the conditions of Lemma 1.1 are fulfilled with $a \in C([0, b] \times$ $[0, b])$ then the estimates (1.4) for the derivatives of the solution $u(t)$ of equation (1.1) can be specified (see [11]).

An effective method for the solution of equations (1.1) with kernels (1.2) is the piecewise collocation method on graded grids. By this method the interval of integration is partitioned into suitable small subintervals and the 
approximate solution is researched in the form of a function which on every subinterval is a polynomial of the same degree. Such a collocation method for equations (1.1) with kernels (1.2) is investigated in [6]. It is shown there how to choose the non-uniform grid so that the method might have the best convergence rate in supremum-norm (see Theorem 2.1 below).

In order to calculate the approximate solution by the piecewise polynomial collocation method it is necessary to solve large linear systems. In the present paper a two-grid iteration scheme is presented for the solution of such systems and fast convergence of this method is shown (see Theorem 3.1 below). Note that similar two-grid iteration methods are considered in $[1-5,9,10,12,13]$.

\section{COLLOCATION METHOD}

Let $N \in \mathbb{N}, r \in \mathbb{R}, r \geq 1$. We introduce in the interval $[0, b]$ the following $4 N+1$ grid points $\left\{t_{j}^{(N)}\right\}$ :

$$
\begin{array}{ll}
t_{j}^{(N)}=\left(\frac{j}{N}\right)^{r} \frac{d}{2}, & j=0,1, \ldots, N ; \\
t_{N+j}^{(N)}=d-t_{N-j}^{(N)}, & j=1, \ldots, N-1 ; \\
t_{2 N+j}^{(N)}=d+\left(\frac{j}{N}\right)^{r} \frac{b-d}{2}, & j=0,1, \ldots, N ; \\
t_{3 N+j}^{(N)}=d+b-t_{3 N-j}^{(N)}, & j=1, \ldots, N .
\end{array}
$$

Here $r \geq 1$ characterizes the degree of the non-uniformity of the grid. If $r=1$ and $d=b / 2$, then the grid points (2.1) are uniformly located in the interval $[0, b]$. If $r>1$ then the grid points (2.1) are more densely located towards the points $0, d$ and $b$.

We determine the collocation points $\left\{\xi_{j, q}^{(N)}\right\}$ in the following way. We choose in the interval $[-1,1] m$ points $\eta_{1}, \ldots, \eta_{m}$,

$$
-1 \leq \eta_{1}<\ldots<\eta_{m} \leq 1,
$$

and set

$$
\xi_{j, q}^{(N)}=t_{j-1}^{(N)}+\frac{\eta_{q}+1}{2}\left(t_{j}^{(N)}-t_{j-1}^{(N)}\right), \quad q=1, \ldots, m ; j=1, \ldots, 4 N .
$$

Note that $\xi_{j, m}^{(N)}=\xi_{j+1,1}^{(N)}=t_{j}^{(N)}$, if $\eta_{1}=-1, \eta_{m}=1(j=1, \ldots, 4 N-1)$.

For a continuous function $u:[0, b] \rightarrow \mathbb{R}$ we construct a piecewise polynomial interpolation function $P_{N} u:[0, b] \rightarrow \mathbb{R}$ as follows: on every interval $\left[t_{j-1}^{(N)}, t_{j}^{(N)}\right]$ $(j=1, \ldots, 4 N) P_{N} u$ is a polynomial of degree not exceeding $m-1$, and

$$
\left(P_{N} u\right)\left(\xi_{j, q}^{(N)}\right)=u\left(\xi_{j, q}^{(N)}\right), \quad q=1, \ldots, m ; j=1, \ldots, 4 N .
$$

Thus, the interpolation function $\left(P_{N} u\right)(t)$ is uniquely defined in every interval $\left[t_{j-1}^{(N)}, t_{j}^{(N)}\right](j=1, \ldots, 4 N)$ separately and may have jumps at $t=t_{j}^{(N)}$, 
$j=1, \ldots, 4 N-1$. If $\eta_{1}=-1, \eta_{m}=1$, then $P_{N} u$ is a continuous function on the interval $[0, b]$. We can define $\left(P_{N} u\right)(t)$ by the formula

$$
\left(P_{N} u\right)(t)=\sum_{q=1}^{m} u\left(\xi_{j, q}^{(N)}\right) \varphi_{j, q}^{(N)}(t), \quad t \in\left[t_{j-1}^{(N)}, t_{j}^{(N)}\right], j=1, \ldots, 4 N
$$

where $\varphi_{j, q}^{(N)}(t), t \in\left[t_{j-1}^{(N)}, t_{j}^{(N)}\right], q=1, \ldots, m ; j=1, \ldots, 4 N$, are the polynomials of degree $m-1$ such that

$$
\varphi_{j, q}^{(N)}\left(\xi_{j, p}^{(N)}\right)=\left\{\begin{array}{ll}
1, & p=q \\
0, & p \neq q
\end{array}\right\}, \quad p, q=1, \ldots, m .
$$

Let us denote by $E_{N}$ the range of the operator $P_{N} \equiv P_{N}^{(m)}, P_{N}: C[0, b] \rightarrow$ $E_{N}$; thus, $E_{N}$ is the space of piecewise polynomial functions $u_{N}$ which on every interval $\left[t_{j-1}^{(N)}, t_{j}^{(N)}\right](j=1, \ldots, 4 N)$ are polynomials of the degree not exceeding $m-1$.

We look for an approximate solution $u_{N} \in E_{N}$ to integral equation (1.1). We require that $u_{N}$ should satisfy the equation (1.1) at the collocation points (2.3):

$$
\begin{gathered}
{\left[u_{N}(t)-\int_{0}^{b} K(t, s) u_{N}(s) d s-f(t)\right]_{t=\xi_{i, p}^{(N)}}=0} \\
p=1, \ldots, m ; \quad i=1, \ldots, 4 N .
\end{gathered}
$$

By the representation (2.5), we can find $u_{N} \in E_{N}$ in the form

$$
u_{N}(t)=\sum_{q=1}^{m} c_{j, q}^{(N)} \varphi_{j, q}^{(N)}(t), \quad t \in\left[t_{j-1}^{(N)}, t_{j}^{(N)}\right], j=1 \ldots, 4 N,
$$

where, as it follows from (2.6),

$$
c_{j, q}^{(N)}=u_{N}\left(\xi_{j, q}^{(N)}\right), \quad q=1, \ldots, m ; j=1, \ldots, 4 N .
$$

Now the collocation conditions (2.7) will take the form of a system which determines the coefficients $c_{i, p}^{(N)}=u_{N}\left(\xi_{i, p}^{(N)}\right)$ :

$$
c_{i, p}^{(N)}=\sum_{j=1}^{4 N} \sum_{q=1}^{m} a_{i, p, j, q}^{(N)} c_{j, q}^{(N)}+f\left(\xi_{i, p}^{(N)}\right), \quad p=1, \ldots, m ; i=1, \ldots, 4 N,
$$

where

$$
\begin{gathered}
a_{i, p, j, q}^{(N)}=\int_{t_{j-1}^{(N)}}^{t_{j}^{(N)}} K\left(\xi_{i, p}^{(N)}, s\right) \varphi_{j, q}^{(N)}(s) d s \\
q=1, \ldots, m ; j=1, \ldots, 4 N ; p=1, \ldots, m ; i=1, \ldots, 4 N .
\end{gathered}
$$


If $\eta_{1}>-1$ or $\eta_{m}<1$, then all collocation points $\xi_{j, q}^{(N)} \quad(q=1, \ldots, m$; $j=1, \ldots 4 N)$ are different and there are $4 m N$ collocation points. In this case the system (2.8) (system (2.7)) has $4 m N=\operatorname{dim} E_{N}$ equations and the same number of unknowns. If $\eta_{1}=-1, \eta_{m}=1$, then part of the collocation points will coincide. The number of different collocation points is $[4 N(m-1)+1]=\operatorname{dim} E_{N}$ and the system (2.8) (system (2.7)) has the same number of equations and unknowns.

On basis of Lemma 1.1 in [6] the following convergence result is proved.

Theorem 2.1. Assume that the following conditions are fulfilled:

1) the kernel (1.2) satisfies the assumptions (A1) and (A2);

2) $f \in E^{\alpha, m}$;

3) the homogeneous integral equation

$$
u(t)=\int_{0}^{b} K(t, s) u(s) d s
$$

has only the trivial solution $u=0$;

4) the collocation points (2.3) with interpolation points (2.2) and grid points (2.1) are used.

Then the equation (1.1) has a unique solution $u^{*}$ and there exists an integer $N_{0} \in \mathbb{N}$ such that, for $N \geq N_{0}$, the collocation conditions (2.7) define a unique approximation $u_{N}^{*} \in E_{N}$ to $u^{*}$. The following error estimates hold:

$$
\sup _{0 \leq t \leq b}\left|u_{N}^{*}(t)-u^{*}(t)\right| \leq c\left\{\begin{array}{llr}
h_{N}^{r(1-\alpha)} & \text { for } & 1 \leq r \leq \frac{m}{1-\alpha} \\
h_{N}^{m} & \text { for } & r \geq \frac{m}{1-\alpha}
\end{array}\right.
$$

where $r$ is the scaling parameter of the grid (2.1), $c$ is a positive constant independent of $N$ (but dependent on $r$ ), and

$$
h_{N}=\max \left\{\frac{d}{2 N}, \frac{b-d}{2 N}\right\}
$$

Remark 2.1. It is shown in [6] that at special collocation points a more rapid convergence (superconvergence) takes place.

To apply the collocation method it is necessary to solve the linear system (2.8). We write this system in the form

$$
\bar{u}_{N}=T_{N} \bar{u}_{N}+\bar{f}_{N}
$$


where

$$
\begin{gathered}
\bar{u}_{N}=\left(c_{1,1}^{(N)}, \ldots, c_{1, m}^{(N)}, c_{2,1}^{(N)}, \ldots, c_{2, m}^{(N)}, \ldots, c_{4 N, 1}^{(N)}, \ldots, c_{4 N, m}^{(N)}\right)^{T}, \\
\bar{f}_{N}=\left(f\left(\xi_{1,1}^{(N)}\right), \ldots, f\left(\xi_{1, m}^{(N)}\right), f\left(\xi_{2,1}^{(N)}\right), \ldots, f\left(\xi_{2, m}^{(N)}\right), \ldots, f\left(\xi_{4 N, 1}^{(N)}\right), \ldots, f\left(\xi_{4 N, m}^{(N)}\right)\right)^{T}
\end{gathered}
$$

are vectors and

$$
T_{N}=\left(a_{i, p, j, q}^{(N)}\right)
$$

is a matrix with elements (2.9) in the following form $\left(a_{i, p, j, q}=a_{i, p, j, q}^{(N)} ; n=\right.$ $4 N)$ :

$$
\left(\begin{array}{cccccccccc}
a_{1,1,1,1} & \ldots & a_{1,1,1, m} & a_{1,1,2,1} & \ldots & a_{1,1,2, m} & \ldots & a_{1,1, n, 1} & \ldots & a_{1,1, n, m} \\
\vdots & & \vdots & \vdots & & \vdots & & \vdots & & \vdots \\
a_{1, m, 1,1} & \ldots & a_{1, m, 1, m} & a_{1, m, 2,1} & \ldots & a_{1, m, 2, m} & \ldots & a_{1, m, n, 1} & \ldots & a_{1, m, n, m} \\
a_{2,1,1,1} & \ldots & a_{2,1,1, m} & a_{2,1,2,1} & \ldots & a_{2,1,2, m} & \ldots & a_{2,1, n, 1} & \ldots & a_{2,1, n, m} \\
\vdots & & \vdots & \vdots & & \vdots & & \vdots & & \vdots \\
a_{2, m, 1,1} & \ldots & a_{2, m, 1, m} & a_{2, m, 2,1} & \ldots & a_{2, m, 2, m} & \ldots & a_{2, m, n, 1} & \ldots & a_{2, m, n, m} \\
\vdots & & \vdots & \vdots & & \vdots & & \vdots & & \vdots \\
a_{n, 1,1,1} & \ldots & a_{n, 1,1, m} & a_{n, 1,2,1} & \ldots & a_{n, 1,2, m} & \ldots & a_{n, 1, n, 1} & \ldots & a_{n, 1, n, m} \\
\vdots & & \vdots & \vdots & & \vdots & & \vdots & & \vdots \\
a_{n, m, 1,1} & \ldots & a_{n, m, 1, m} & a_{n, m, 2,1} & \ldots & a_{n, m, 2, m} & \ldots & a_{n, m, n, 1} & \ldots & a_{n, m, n, m}
\end{array}\right)
$$

Usually the number of equations in (2.13) is large and, as a result of this, direct solving of (2.13) is rather complicated. An effective method for solving this system is a two-grid iteration method.

\section{TWO-GRID METHOD}

In addition to the original grid corresponding to $N \in \mathbb{N}$ (see (2.1)), we define by (2.1) the coarse grid, corresponding to an integer $M \in \mathbb{N}, M<N$. More precisely, we choose $N$ and $M$ so that $N / M$ is an integer greater than 1 . Then every subinterval $\left[t_{j-1}^{(N)}, t_{j}^{(N)}\right](j=1, \ldots, 4 N)$ is fully contained in some subinterval $\left[t_{i-1}^{(M)}, t_{i}^{(M)}\right](i=1, \ldots, 4 M)$ of the coarse grid.

For solving the system (2.13) the following two-grid iteration method is used:

$$
\left\{\begin{array}{l}
\bar{v}_{N}^{l}=\bar{u}_{N}^{l}-T_{N} \bar{u}_{N}^{l}-\bar{f}_{N}, \\
\bar{w}_{M}^{l}=\left(I_{M}-T_{M}\right)^{-1} R_{N, M} T_{N} \bar{v}_{N}^{l}, \\
\bar{u}_{N}^{+1}=\bar{u}_{N}^{l}-\bar{v}_{N}^{l}-Q_{M, N} \bar{w}_{M}^{l}, \quad l=0,1, \ldots,
\end{array}\right.
$$

where $\bar{u}_{N}^{0}$ is the initial guess of $\bar{u}_{N}, I_{M}$ is the identity matrix, $R_{N, M}: \mathbb{R}^{d_{N}} \rightarrow \mathbb{R}^{d_{M}}$ 
$\left(d_{N}=\operatorname{dim} E_{N}, d_{M}=\operatorname{dim} E_{M}\right)$ is the restriction operator defined by

$$
\begin{aligned}
\left(R_{N, M} T_{N} \bar{v}_{N}^{l}\right)\left(\xi_{i, p}^{(M)}\right) & =\sum_{j=1}^{4 N} \sum_{q=1}^{m}\left(\int_{t_{j-1}^{(N)}}^{t_{j}^{(N)}} K\left(\xi_{i, p}^{(M)}, s\right) \varphi_{j, q}^{(N)}(s) d s\right) v_{N}^{l}\left(\xi_{j, q}^{(N)}\right), \\
p & =1, \ldots, m ; \quad i=1, \ldots, 4 M
\end{aligned}
$$

and $Q_{M, N}: \mathbb{R}^{d_{M}} \rightarrow \mathbb{R}^{d_{N}}$ is the prolongation operator defined by

$$
\begin{gathered}
\left(Q_{M, N} \bar{w}_{M}^{l}\right)\left(\xi_{j, q}^{(N)}\right)=\sum_{p=1}^{m} w_{m}^{l}\left(\xi_{i, p}^{(M)}\right) \varphi_{i, p}^{(M)}\left(\xi_{j, q}^{(N)}\right) \text { if } \xi_{j, q}^{(N)} \in\left[t_{i-1}^{(M)}, t_{i}^{(M)}\right] \\
i=1, \ldots, 4 M, \quad q=1, \ldots, m ; \quad j=1, \ldots 4 N .
\end{gathered}
$$

Here $v_{N}^{l}\left(\xi_{j, q}^{(N)}\right),\left(R_{N, M} T_{N} \bar{v}_{N}^{l}\right)\left(\xi_{i, p}^{(M)}\right), w_{M}^{l}\left(\xi_{i, p}^{(M)}\right)$ and $\left(Q_{M, N} \bar{w}_{M}^{l}\right)\left(\xi_{j, q}^{(N)}\right)$ are the corresponding components of the vectors $\bar{v}_{N}^{l}, R_{N, M} T_{N} \bar{v}_{N}^{l}, \bar{w}_{M}^{l}$ and $Q_{M, N} \bar{w}_{M}$, respectively. The ordering of the components of these vectors is the same as that one for the vectors $\bar{u}_{N}$ and $\bar{f}_{N}$ above (see (2.13)).

Similar two-grid iteration methods are considered in $[1-5,9,10,12,13]$. Our treatment will follow the approach of [10].

We write the integral equation in the form

$$
u=T u+f
$$

where

$$
(T u)(t)=\int_{0}^{b} K(t, s) u(s) d s .
$$

The collocation conditions (2.7) are equivalent to the operator equation

$$
u_{N}=P_{N} T u_{N}+P_{N} f
$$

with operators $P_{N}: C[0, b] \rightarrow E_{N}$, introduced in Section 2. Using (A1) and (A2) we can establish the following result.

Lemma 3.1. Let the conditions (A1) and (A2) about the kernel (1.2) hold, and let the collocation points (2.3) with grid points (2.1) be used. Then for every choice of collocation parameters (2.2),

$$
\left\|T-P_{N} T\right\|_{L^{\infty}(0, b) \rightarrow L^{\infty}(0, b)} \leq c h_{N}^{1-\alpha},
$$

where $h_{N}$ is defined in (2.12) and the constant $c$ is independent of $N$.

In order to give to the method (3.1) a more convenient form for convergence analysis, we introduce the operators $R_{\infty, N}: E_{N} \rightarrow \mathbb{R}^{d_{N}}$ and $Q_{N, \infty}: \mathbb{R}^{d_{N}} \rightarrow E_{N}$ by the following equalities:

$$
R_{\infty, N} u=\left(u\left(\xi_{1,1}^{(N)}\right), \ldots, u\left(\xi_{1, m}^{(N)}\right), u\left(\xi_{2,1}^{(N)}\right), \ldots, u\left(\xi_{2, m}^{(N)}\right), \ldots, u\left(\xi_{4 N, 1}^{(N)}\right), \ldots, u\left(\xi_{4 N, m}^{(N)}\right)\right)
$$


for $u \in E_{N}$, and

$$
\left(Q_{N, \infty} \bar{u}_{N}\right)(t)=\sum_{q=1}^{m} u_{N}\left(\xi_{j, q}^{(N)}\right) \varphi_{j, q}^{(N)}(t), \quad t \in\left[t_{j-1}^{(N)}, t_{j}^{(N)}\right], j=1, \ldots, 4 N
$$

for

$$
\bar{u}_{N}=\left(u_{N}\left(\xi_{1,1}^{(N)}\right), \ldots, u_{N}\left(\xi_{1, m}^{(N)}\right), \ldots, u_{N}\left(\xi_{4 N, 1}^{(N)}\right), \ldots, u_{N}\left(\xi_{4 N, m}^{(N)}\right)\right) \in \mathbb{R}^{d_{N}}
$$

Actually, we shall use the definition (3.8) for applying $R_{\infty, N}$ to all functions $u(t)$ which are defined at $t=\xi_{j, q}^{(N)}, q=1, \ldots, m ; j=1, \ldots, 4 N$. For later use, introduce

$$
u_{N}^{l}=Q_{N, \infty} \bar{u}_{N}^{l}, v_{N}^{l}=Q_{N, \infty} \bar{v}_{N}^{l}, w_{M}^{l}=Q_{M, \infty} \bar{w}_{M}^{l},
$$

where $l=0,1, \ldots$, and $\bar{w}_{N}^{l}$ and $\bar{u}_{N}^{l+1}$ are determined by (3.1) for a initial guess $\bar{u}_{N}^{0} \in \mathbb{R}^{d_{N}}$. Then

$$
\begin{array}{cl}
\bar{u}_{N}^{l}=R_{\infty, N} u_{N}^{l}, \quad \bar{v}_{N}^{l}=R_{\infty, N} v_{N}^{l}, \quad \bar{w}_{M}^{l}=R_{\infty, M} w_{M}^{l}, \\
R_{\infty, N} Q_{N, \infty}=I_{N}, \quad Q_{N, \infty} R_{\infty, N}=P_{N} \\
R_{\infty, N} T Q_{N, \infty}=T_{N}, \quad Q_{N, \infty} Q_{M, N}=Q_{M, \infty}
\end{array}
$$

Using these notations and identies we can rewrite formulas (3.1) as follows:

$$
\left\{\begin{array}{l}
v_{N}^{l}=u_{N}^{l}-P_{N} T u_{N}^{l}-P_{N} f \\
w_{M}^{l}=P_{M} T w_{M}^{l}+P_{M} T v_{N}^{l} \\
u_{N}^{l+1}=u_{N}^{l}-v_{N}^{l}-w_{M}^{l}, \quad l=0,1, \ldots
\end{array}\right.
$$

Whereas $u_{N}^{0}=Q_{N, \infty} \bar{u}_{N}^{0} \in E_{N}$ we also have $v_{N}^{l} \in E_{N}, w_{M}^{l} \in E_{M} \subset E_{N}$ and $u_{N}^{l+1} \in E_{N}, l=0,1, \ldots$ Therefore the methods (3.1) and (3.9) are equivalent. At the same time the method (3.9) is an iteration method to solve (3.6).

We are now ready to prove the following result about the convergence of the two-grid method (3.1).

Theorem 3.1. Let the assumptions of Theorem 1.1 hold. Then there exists an integer $M_{0}>0$ such that, for $N \geq M_{0}$, the system (2.13) has a unique solution $\bar{u}_{N, 0}$. The two-grid iteration method (3.1) convergences to this solution for $M \geq M_{0}, N / M=2,3, \ldots$, and for every choice of the initial guess $\bar{u}_{N}^{0}$ to:

$$
\left\|\bar{u}_{N}^{l+1}-\bar{u}_{N, 0}\right\|_{\infty} \leq \mathrm{const} h_{M}^{1-\alpha}\left\|\bar{u}_{N}^{l}-\bar{u}_{N, 0}\right\|_{\infty}, \quad l=0,1, \ldots,
$$

where $h_{M}=\max \{d / 2 M,(b-d) / 2 M\}$ and

$$
\left\|\bar{u}_{N}\right\|_{\infty}=\max _{j=1, \ldots, 4 N ; q=1, \ldots, m}\left|u_{N}\left(\xi_{j, q}^{(N)}\right)\right| .
$$


Proof. It follows from (A1) and (A2) that the integral operator (3.5) with the kernel $(1.2)$ is a compact operator on $L^{\infty}(0, b)$ to $L^{\infty}(0, b)$ [7]. As the homogeneous equation $u=T u$ has only the trivial solution $u=0$, the operator $I-T: L^{\infty}(0, b) \rightarrow$ $L^{\infty}(0, b)$, with the identity operator I, has a bounded inverse $(I-T)^{-1}: L^{\infty}(0, b) \rightarrow$ $L^{\infty}(0, b)$. By Lemma 3.1, $\left\|T-P_{M} T\right\|_{L^{\infty}(0, b) \rightarrow L^{\infty}(0, b)} \rightarrow 0$, if $h_{M} \rightarrow 0$. Therefore there occurs $M_{0}>0$ such that for all $M \geq M_{0},\left(I-P_{M} T\right)^{-1}: L^{\infty}(0, b) \rightarrow L^{\infty}(0, b)$ exist and their norms are uniformly bounded:

$$
\left\|\left(I-P_{M} T\right)^{-1}\right\|_{L^{\infty}(0, b) \rightarrow L^{\infty}(0, b)} \leq \mathrm{const} \quad\left(M \geq M_{0}\right) .
$$

It follows from this that (2.13) has a unique solution $u_{N, 0} \in E_{N}$ for every $N \geq M_{0}$. Consequently, for $M \geq M_{0}, N / M=2,3, \ldots$, and for a initial guess $u_{N}^{0} \in E_{N}$ to $u_{N, 0}$, the formulas (3.9) define a sequence of elements $u_{N}^{l} \in E_{N}, l=1,2, \ldots$, and

$$
u_{N}^{l+1}-u_{N, 0}=\left(I-P_{M} T\right)^{-1}\left(P_{N}-P_{M}\right) T\left(u_{N}^{l}-u_{N, 0}\right), \quad l=0,1, \ldots
$$

Indeed, we have

$$
\begin{aligned}
& \left(I-P_{M} T\right)\left(u_{N}^{l+1}-u_{N, 0}\right)=\left(I-P_{M} T\right)\left[u_{N}^{l}-v_{N}^{l}-w_{M}^{l}-u_{N, 0}\right]= \\
& =\left(I-P_{M} T\right)\left[P_{N} T u_{N}^{l}+P_{N} f-\left(I-P_{M} T\right)^{-1} P_{M} T v_{N}^{l}-u_{N, 0}\right]= \\
& =P_{N} T u_{N}^{l}+P_{N} f-P_{M} T P_{N} T u_{N}^{l}-P_{M} T P_{N} f-P_{M} T v_{N}^{l}-u_{N, 0}+P_{M} T u_{N, 0}= \\
& =P_{N} T u_{N}^{l}+u_{N, 0}-P_{N} T u_{N, 0}-P_{M} T P_{N} T u_{N}^{l}-P_{M} T P_{N} f-P M T u_{N}^{l}+ \\
& +P_{M} T P_{N} T u_{N}^{l}+P_{M} T P_{N} f-u_{N, 0}+P_{M} T u_{N, 0}= \\
& =P_{N} T\left(u_{N}^{l}-u_{N, 0}\right)-P_{M} T\left(u_{N}^{l}-u_{N, 0}\right)= \\
& =\left(P_{N}-P_{M}\right) T\left(u_{N}^{l}-u_{N, 0}\right) .
\end{aligned}
$$

Applying $\left(I-P_{M} T\right)^{-1}$ to the identity $\left(I-P_{M} T\right)\left(u_{N}^{l+1}-u_{N, 0}\right)=\left(P_{N}-P_{M}\right)\left(T\left(u_{N}^{l}-\right.\right.$ $\left.u_{N, 0}\right)$, we obtain (3.13). Further, using Lemma 3.1,

$$
\begin{gathered}
\left\|\left(P_{N}-P_{M}\right) T\right\|_{L^{\infty}(0, b) \rightarrow L^{\infty}(0, b)} \leq\left\|T-P_{N} T\right\|_{L^{\infty}(0, b) \rightarrow L^{\infty}(0, b)}+ \\
+\left\|T-P_{M} T\right\|_{L^{\infty}(0, b) \rightarrow L^{\infty}(0, b)} \leq c\left(h_{N}^{1-\alpha}+h_{M}^{1-\alpha}\right) \leq c^{\prime} h_{M}^{1-\alpha},
\end{gathered}
$$

with suitable constants $c$ and $c^{\prime}$. Combining this and (3.13) and (3.12) for $M \geq M_{0}$, $N / M=2,3, \ldots$, we have

$$
\left\|u_{N}^{l+1}-u_{N, 0}\right\|_{L^{\infty}(0, b)} \leq c^{\prime \prime} h_{M}^{1-\alpha}\left\|u_{N}^{l}-u_{N, 0}\right\|_{L^{\infty}(0, b)}, \quad l=0,1, \ldots
$$

with a constant $c^{\prime \prime}$ which is independent of $M, N$ and $l$. The estimate (3.10) now follows from (3.14) because

$$
\left\|\bar{u}_{N}^{l+1}-\bar{u}_{N, 0}\right\|_{\infty}=\left\|R_{\infty, N}\left(u_{N}^{l+1}-u_{N, 0}\right)\right\|_{\infty} \leq\left\|u_{N}^{l+1}-u_{N, 0}\right\|_{\infty}
$$

and

$$
\left\|u_{N}^{l}-u_{N, 0}\right\|_{\infty}=\left\|P_{N, \infty}\left(\bar{u}_{N}^{l}-u_{N, 0}\right)\right\|_{\infty} \leq c^{\prime \prime \prime}\left\|\bar{u}_{N}^{l}-\bar{u}_{N, 0}\right\|_{\infty},
$$

with a constant $c^{\prime \prime \prime}$ which is independent of $N$. 


\section{REFERENCES}

[1] K. E. Atkinson. A survey of numerical methods for solving nonlinear integral equations. J. Integral Equations Appl., 4 1992, 15 - 46.

[2] K. E. Atkinson. Two-grid iteration methods for linear integral equations of the second kind on piecewise smooth surfaces in $R^{3}$. SIAM J. Sci. Comput., 15 1994, $1083-1104$.

[3] K. E. Atkinson. The Numerical Solution of Integral Equations of the Second Kind. Cambridge University Press, 1997.

[4] W. Hackbusch. Multi-Grid Methods and Applications. Springer Verlag, Berlin, 1985.

[5] W. Hackbusch. Integralgleichungen. Theorie und Numerik. Teubner, Stuttgart, 1989. English translation: Integral Equation. Theory and Numerical Methods. ISNM 120, Birkhäuser, Basel, 1995

[6] K. Hakk, A. Pedas. Numerical solutions and their superconvergence for weakly singular integral equations with discontinuous coefficients. Mathematical Modelling and Analysis, (R. Ciegis ed.), Vilnius "Technika", 3 1998, $104-113$.

[7] L. V. Kantorovitch, G. P. Akilov. Functional Analysis. Nauka, Moscow, 1977. (in Russian)

[8] A. Pedas, G. Vainikko. The smoothness of solutions to nonlinear weakly singular integral equations. Z. Anal. Anwendungen, 13 1994, 463 - 467.

[9] E. Tamme. Two-grid method for nonlinear multi-dimensional weakly singular integral equations. J. Integral Equations Appl., 7 1995, 99 - 113.

[10] E. Tamme. Two-grid method for the solution of weakly singular integral equations by piecewise polynomial approximation. Proc. Estonian Acad. Sci. Phys. Math., 46 1997, $241-250$.

[11] P. Uba. Smoothness of the solution of the weakly singular integral equation with discontinuous coefficient. Proc. Estonian Acad. Sci. Phys. Math., 37 (2), 1988, 192 203.(in Russian)

[12] G. Vainikko. Multidimensional weakly singular integral equations. Springer Verlag, Berlin, 1993.

[13] G. Vainikko, A. Pedas, P. Uba. Methods for Solving Weakly Singular Integral Equations. Univ. of Tartu, Tartu, 1984. (in Russian)

\section{SILPNAI SINGULIARIUU INTEGRALINIŲ LYGČIŲ SPRENDIMAS DVITINKLIU ITERACINIU METODU}

K. Hakk, A. Pedas

Sprendžiant silpnai singuliarias integralines lygtis gabalais polinominiu kolokaciju metodu tenka spręsti didelès dimensijos tiesinių lygčiuc sistemas. Šiame darbe tokios sistemos sprendžiamos dvitinkliu iteraciniu metodu. Ištirtas iteracinio metodo konvergavimas, gauti konvergavimo greičio įverčiai. 